\title{
Neopterin and kynurenic acid as predictors of stroke recurrence and mortality: a multicentre prospective cohort study on biomarkers of inflammation measured three months after ischemic stroke
}

Katinka Nordheim Alme ${ }^{1,2^{*}}$, Arve Ulvik ${ }^{3}$, Torunn Askim ${ }^{4}$, Jörg Assmus ${ }^{5}$, Tom Eirik Mollnes ${ }^{6,7,8}$, Mala Naik 2,9, Halvor Næss ${ }^{1,10,11}$, Ingvild Saltvedt ${ }^{4,12}$, Per-Magne Ueland ${ }^{3}$ and Anne-Brita Knapskog ${ }^{13}$

\begin{abstract}
Background: Chronic low-grade inflammation is associated with both ischemic stroke and sedentary behaviour. The aim of this study was to investigate the predictive abilities of biomarkers of inflammation and immune modulation associated with sedentary behaviour for ischemic stroke recurrence and mortality in a stroke population.

Methods: Patients admitted to hospital for acute stroke were recruited to the prospective multicentre cohort study, the Norwegian Cognitive Impairment After Stroke (Nor-COAST) study, from May 2015 until March 2017. Patients with ischemic stroke, blood samples available from the three-month follow-up, and no stroke recurrence before the threemonth follow-up were included. Serum was analysed for C-reactive protein (CRP) with high-sensitive technique, and plasma for interleukin-6 (IL-6), neopterin, pyridoxic acid ratio index (PAr-index: 4-pyridoxic acid: [pyrioxal+pyridoxal-5'phosphate]) and kynurenic acid (KA). Ischemic stroke recurrence and death were identified by the Norwegian Stroke Registry and the Cause of Death Registry until 31 December 2018.

Results: The study included 354 patients, 57\% male, mean age 73 (SD 11) years, mean observation time 2.5 (SD 0.6) years, and median National Institute of Health Stroke Scale of 0 (IQR 1) at three months. CRP was associated with mortality (HR 1.40, Cl 1.01, 1.96, $p=0.046$ ), and neopterin was associated with the combined endpoint (recurrent ischemic stroke or death) (HR 1.52, Cl 1.06, 2.20, $p=0.023$ ), adjusted for age, sex, prior cerebrovascular disease, modified Rankin Scale, and creatinine. When adding neopterin and KA to the same model, KA was negatively associated $(\mathrm{HR} 0.57, \mathrm{Cl}$ $0.33,0.97, p=0.038$ ), and neopterin was positively associated ( $\mathrm{HR} 1.61, \mathrm{Cl} 1.02,2.54, p=0.040$ ) with mortality. Patients with cardioembolic stroke at baseline had higher levels of inflammation at three months.
\end{abstract}

Conclusion: Neopterin might be a valuable prognostic biomarker in stroke patients. The use of KA as a measure of anti-inflammatory capacity should be investigated further.

Trial registration: The study was registered at Clinicaltrials.gov (NCT02650531). First posted on 08/01/2016.

Keywords: Sedentary behaviour, Inflammation, Immune modulation, Vascular disease, Kynurenine pathway, Stroke

*Correspondence: Katinka.alme@gmail.com

1 Institute of Clinical Medicine (K1), University of Bergen, Bergen, Norway

Full list of author information is available at the end of the article

(c) The Author(s) 2021. Open Access This article is licensed under a Creative Commons Attribution 4.0 International License, which permits use, sharing, adaptation, distribution and reproduction in any medium or format, as long as you give appropriate credit to the original author(s) and the source, provide a link to the Creative Commons licence, and indicate if changes were made. The images or other third party material in this article are included in the article's Creative Commons licence, unless indicated otherwise in a credit line to the material. If material is not included in the article's Creative Commons licence and your intended use is not permitted by statutory regulation or exceeds the permitted use, you will need to obtain permission directly from the copyright holder. To view a copy of this licence, visit http://creativecommons.org/licenses/by/4.0/. The Creative Commons Public Domain Dedication waiver (http://creativeco mmons.org/publicdomain/zero/1.0/) applies to the data made available in this article, unless otherwise stated in a credit line to the data. 


\section{Background}

Ischemic stroke is associated with chronic low-grade inflammation, and there is a need for more knowledge about mechanisms and management [1-4]. Prognosis, outcomes, and management of stroke depends on stroke subtype, defined by the assumed aetiology of atherosclerosis, cardioembolism, or small vessel disease [5, 6]. In the context of inflammation, atherosclerosis is the most studied subtype, but inflammation has been found to contribute to all three categories [7-10]. Despite the evidence, clinical trials using drugs targeting inflammation have so far been inconclusive, and there are to date no established preventive treatment strategies that target inflammation in particular $[4,11]$, possibly related to the challenge of obtaining an optimal balance between potential benefits and an increased risk of (fatal) infections [4]. Obviously, treatment strategies that minimise severe side effects are desired.

Reducing chronic low-grade inflammation by targeting sedentary behaviour has shown promising results [12]. Still, there is a need for valid biomarkers associated with inflammation with explanatory and predictive properties for outcomes like vascular disease and mortality [3]. In a prior study, we investigated blood biomarkers of inflammation and immune modulation in a stroke population measured 3 months after the acute ischemic event and their association to objectively measured sedentary time. We identified the biomarkers C-reactive protein (CRP), interleukin-6 (IL-6), neopterin, and the pyridoxal acid ratio-index (PAr-index) to be positively associated, and kynurenic acid (KA) to be negatively associated with objectively measured sedentary behaviour [13]. CRP [14], IL-6 [15], neopterin [16, 17] and PArindex [18] are inflammatory biomarkers associated with vascular disease. KA is part of a negative feedback loop inducing immune tolerance $[19,20]$. Hence, even though it will rise in response to inflammation, this response is believed to be beneficial for disease progression during an inflammatory state [20]. The association to vascular disease progression is unclear.

In this study, we investigated the associations between biomarkers of inflammation and stroke recurrence and all-cause mortality. Finally, given the above-mentioned clinical importance of the stroke subtypes, we also investigated the association to the stroke subtype at baseline.

\section{Material and methods}

\section{Subjects}

The patients were included in the prospective multicentre cohort study, the Norwegian Cognitive Impairment After Stroke (Nor-COAST) study, at admission for acute stroke between May 2015 and March 2017. The inclusion criteria were 1) hospital admission to one of the five participating hospitals, 2) acute stroke following the World Health Organisation definition or finding of acute stroke on imaging 3) being able to communicate in one of the Scandinavian languages, 4) above 18 years of age, 5) living in the catchment area. Patients were excluded if they had a life expectancy of less than 3 months. Details about the Nor-COAST population are described in prior studies [21, 22]. For this study, only patients with ischemic stroke at baseline who participated at the threemonth follow-up and with data on at least one of the relevant biomarkers were included. To avoid the acute inflammatory response associated with the acute stroke event, we chose the three-month follow-up after the acute stroke as the baseline for this study, assuming the inflammatory biomarkers were not affected by the acute event at this time point (Fig. 1) [23].

\section{Clinical data}

Demographic data and information about stroke properties, namely lesion type and stroke subtype, and stroke risk factors, were collected at baseline. Waist circumference, smoking status, stroke severity and functional outcomes were assessed at the three-month follow-up. Stroke severity was measured using the National Institute of Health Stroke Scale (NIHSS), global function by use of the modified Rankin Scale (mRS), and basic activities of daily living (ADL) by the Barthel Index (BI). The diagnosis of diabetes mellitus was defined at baseline by medical history and/or medication use (Anatomical therapeutical chemical Classification (ATC): A10) and/or HbA1c $\geq 6.5 \%$ at baseline. Hypercholesterolemia at baseline was defined by medication use (ATC: C10) and/or total cholesterol $>6.2 \mathrm{mmol} / \mathrm{L}$ and/or $\mathrm{LDL} \geq 4.1 \mathrm{mmol} / \mathrm{L}$ at baseline. Hypertension at baseline was defined by medication use (ATC: C02, C03, C04, C07, C08, C09).

\section{Laboratory analyses}

At the three-month follow-up, non-fasting blood samples were collected for routine analysis and for storage in the biobank.

Routine clinical-chemical analyses: serum C-reactive protein (CRP) - measured using a high-sensitive technique $(\mathrm{mg} / \mathrm{L})-$ and serum creatinine $(\mu \mathrm{mol} / \mathrm{L})$ were analysed at the local laboratories of the inclusion centres.

Sample collection of research analyses: Aliquots of serum and plasma were immediately frozen at $-80^{\circ} \mathrm{C}$, and sent on dry ice to BioBank1, Central Norway Regional Health Authority for storage. The inflammatory biomarkers were analysed in 2019. Two aliquots of plasma were used, one for each of the two laboratories. The samples were thawed only once. The selection of biomarkers for this study was based on prior findings [13] and included CRP (mg/L), interleukin-6 (IL-6, pg/ 


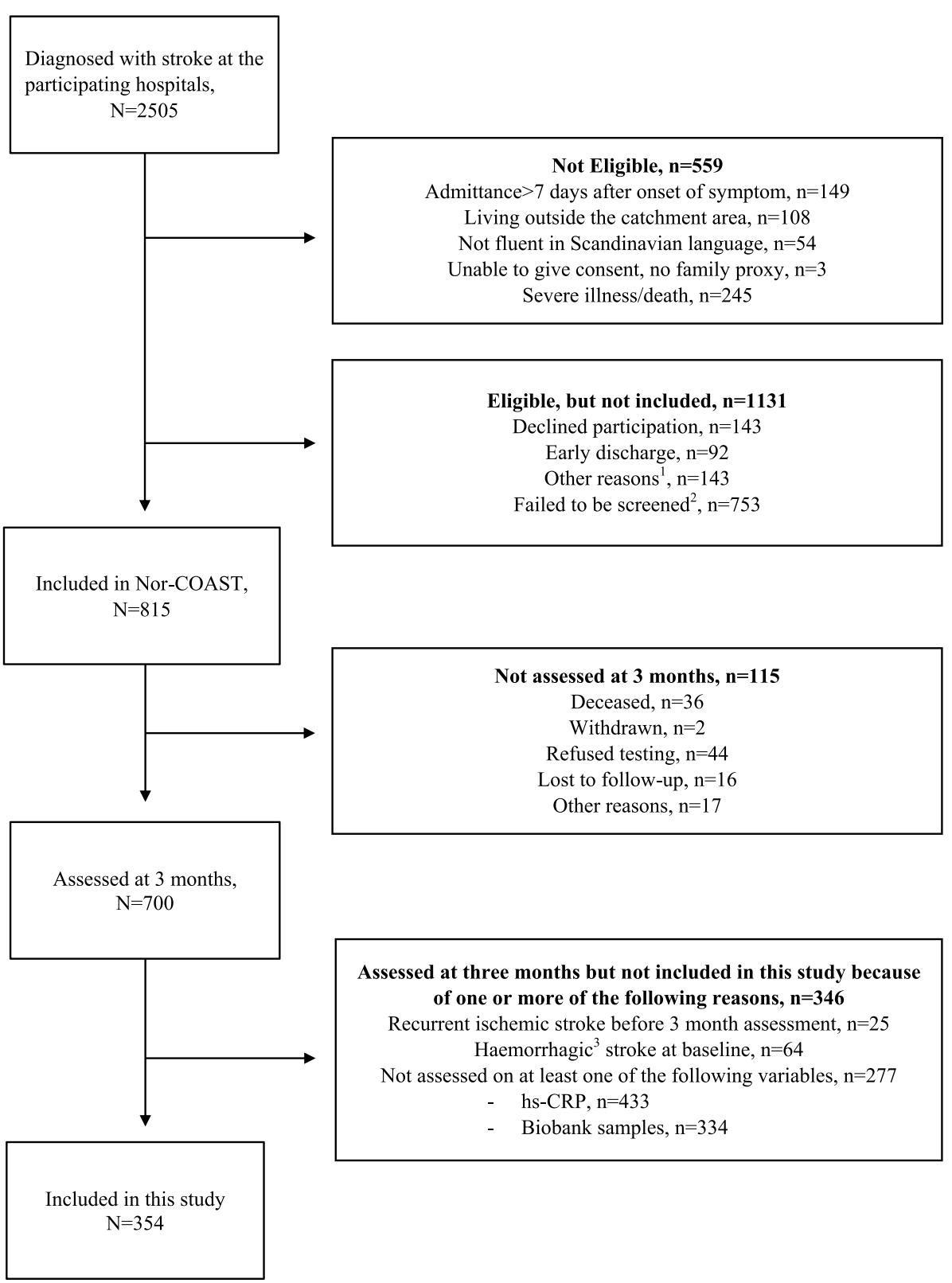

Fig. 1 Patient selection. ${ }^{1}$ Other reasons: i.e.: delirious patient, hearing, uncertainty about the diagnosis, multi morbid, nursing home resident. ${ }^{2}$ Failed to screen: infrastructure on the ward, vacation/weekends. ${ }^{3}$ Not haemorragic transformation

$\mathrm{ml})$, neopterin (nmol/L), 4-pyridoxic acid (nmol/L), pyridoxal, pyridoxal 5'-phosphate (nmol/L), and kynurenic acid (KA, nmol/L). The pyridoxic acid ratio index - PArindex: 4-pyridoxic acid (pyrioxal+pyridoxal $5^{\prime}$-phosphate) [24] was calculated. The cytokines were analysed at Research Laboratory Nordland Hospital using the BioPlex technology kits obtained from Bio-Rad Laboratories Inc., Hercules, CA.. The other biomarkers were analysed as part of analytic platform D at Bevital A/S (Bergen, Norway) by liquid chromatography/tandem mass spectrometry. Because of the sample size, IL-6 was analysed using multiple trays. The upper and lower detection limit varied among the trays according to the standard curve. There were no values above or below the detection limits for IL-6. For the biomarkers from BeVital AS (neopterin, kynurenic acid, and the B6 vitamers), the performance of the method has been published previously [25]. There were no values out of range. 


\section{Outcomes}

Recurrent ischemic stroke was identified by the Norwegian Stroke Registry. The coverage was $84-87 \%$ in the period from 2015 to 2018, and stroke was defined according to the WHO's definition [26]. Death was identified by the Norwegian Cause of Death Registry, which has a general coverage of $98 \%$ of deaths of Norwegian citizens within the country or abroad [27]. The data extraction was performed on 31 December 2018.

Patient outcomes were defined based on the first event: recurrent ischemic stroke, death or no event. Some of the patients died during the follow-up period after first having a stroke recurrence. In the regression analyses, these patients were included in both groups. A third outcome was defined as the composite of recurrent ischemic stroke and death. The patients with both outcomes were counted only once.

\section{Statistics}

For the baseline and three-month descriptions, patients were stratified by first event and analysed using the chisquared test for categorical variables (numbers and percentages), one-way ANOVA for continuous variables, and Kruskal-Wallis $\mathrm{H}$-test for the continuous variables that were not normally distributed. Data were presented as means with standard deviations (SD) and medians with interquartile range (IQR).

One-way ANOVA were used for the analyses of laboratory values according to stroke subtype at baseline. The subgroup "other determined" was excluded.

The associations between the individual biomarkers and the outcomes stroke, mortality and the composite endpoint were analysed using Cox regressions. The start of the follow-up time was defined as the date of the three-month visit to the hospital. For the outcome "recurrent ischemic stroke", an additional analysis using a competing-risk Cox regression based on the method of Fine and Grey [28] as an alternative to the regular Cox regression was used [29], with death as a competing outcome for ischemic stroke. All the biomarkers were right-skewed. After log-transformation, regression residuals approached normal distribution. The variables were standardised, and the hazard ratios are given per standard deviation change of the variable value. To account for potential confounders, we added modified Rankin scale and prior cerebrovascular disease as independent variables, because they have been found to be associated with both stroke recurrence [30] and with inflammation (mRS [31], prior cerebrovascular disease (before the baseline stroke in this study) [32]). We also added age and sex. Creatinin was included because some of the biomarkers had renal clearance (Model 1). As the blood samples were taken at the three-month follow-up, we used the results of the mRS recorded at this time point. To account for the different pathophysiology of the stroke subtypes, we did a second analysis where we added the Trial of Org 10,172 in Acute Stroke Treatment (TOAST) criteria to the model (Model 2), defining five stroke subtypes (large artery atherosclerosis, cardioembolic, cerebral small vessel disease, other determined, undetermined). The stroke subtype "other determined" is used when the cause of stroke is determined (i.e. dissection, vasculitis). This group was excluded from this analysis because of low sample size. Because KA is part of a feedback loop in response to inflammation, a third regression model including both KA and neopterin in addition to the same covariates as in Model 1 was made.

The significance level was set to 0.05 , but $p$-values $>0.01$ should be evaluated with caution due to a high number of tests. The analyses were performed in STATA/SE 16.1 (Stata Corp LLC, College Station, TX, USA).

\section{Results}

Patient characteristics and laboratory values

Of the 700 patients attending the three-month followup, 354 were included in this sub-study. The remaining 346 patients were excluded due to at least one of the following reasons: stroke before 3 months $(n=25)$, haemorrhagic stroke at baseline $(n=64)$, or no relevant biomarker available $(n=277)$.

The mean follow-up time from baseline for the whole group was 2.5 (0.6) years, and the mean time-to-event was 1.5 (1.0) and $1.6(0.7)$ years for stroke and death, respectively. From the three-month follow-up and throughout the follow-up time, 16 (4.5\%) patients had ischemic stroke recurrence. In total, 28 (7.9\%) patients died, and 25 (7.1\%) of them died without being registered as having stroke recurrence.

The baseline and three-month data are presented in Table 1, stratified by the first event. The patients who died were older compared to those with no event. Patients with no events had a higher Barthels Index ( $\mathrm{p}$ $0.007)$ and a lower mRS $(p<0.001)$. There were overall differences between the groups in the presence of prior ischemic stroke $(\mathrm{p}<0.001)$, prior coronary artery disease $(p=0.007)$, and atrial fibrillation at baseline $(p=0.034)$, the patients experiencing no events showing the lowest prevalence. There were overall differences between the groups in the values for IL-6, neopterin, and the PAr-index, where the patients who died had the highest values.

\section{Stroke subtype}

The biomarker levels by stroke subtype at baseline are presented in Table 2. There was a significant overall 
Table 1 Baseline and three-month characteristics according to the first event after three-month follow-up

\begin{tabular}{|c|c|c|c|c|c|}
\hline & $\begin{array}{l}\text { All } \\
N=354\end{array}$ & $\begin{array}{l}\text { New IS } \\
N=16\end{array}$ & $\begin{array}{l}\text { Death } \\
N=25^{1}\end{array}$ & $\begin{array}{l}\text { No event } \\
N=313\end{array}$ & $P$-value \\
\hline $\mathrm{Age}^{2,3}$, mean years $(\mathrm{SD})$ & $73(11)$ & $73(10)$ & $82(9)$ & $73(11)$ & $<0.001$ \\
\hline Male $^{4}$ sex, n (\%) & $201(57)$ & $11(69)$ & $16(64)$ & $174(56)$ & 0.439 \\
\hline Observation time ${ }^{3}$, mean years (SD) & $2.5(6)$ & $1.5(1.0)$ & $1.6(0.7)$ & $2.6(0.5)$ & $<0.001$ \\
\hline \multicolumn{6}{|l|}{ Baseline } \\
\hline TOAST $^{4}$ subtype ${ }^{6}, \mathrm{n}(\%)$ & & & & & 0.661 \\
\hline Large artery & $30(9)$ & $1(6)$ & 0 & $29(10)$ & \\
\hline Cardioembolic & $72(21)$ & $5(31)$ & $6(24)$ & $61(20)$ & \\
\hline Small vessel disease & $83(24)$ & $4(25)$ & $6(7)$ & $73(24)$ & \\
\hline Other determined & $10(3)$ & $1(6)$ & 0 & $9(3)$ & \\
\hline Undetermined & $128(43)$ & $5(31)$ & $13(52)$ & $128(43)$ & \\
\hline Prior $I^{4}, \mathrm{n}(\%)$ & $72(20)$ & $7(44)$ & $13(52)$ & $52(17)$ & $<0.001$ \\
\hline Prior $\mathrm{CHD}^{4}, \mathrm{n}(\%)$ & $61(17)$ & $3(19)$ & $10(40)$ & $48(13)$ & 0.007 \\
\hline Atrial fibrillation ${ }^{4}, \mathrm{n}(\%)$ & $89(25)$ & $6(37)$ & $11(44)$ & $72(23)$ & 0.034 \\
\hline Hypertension ${ }^{4}, \mathrm{n}(\%)$ & $202(57)$ & $11(69)$ & $19(76)$ & $172(60)$ & 0.077 \\
\hline Hypercholesterolemia ${ }^{4}, \mathrm{n}(\%)$ & $178(50)$ & $8(50)$ & $16(64)$ & $154(46)$ & 0363 \\
\hline Diabetes mellitus ${ }^{4}$ n (\%) & $60(17.0)$ & 0 & $6(24)$ & $54(17)$ & 0.124 \\
\hline \multicolumn{6}{|l|}{ Three-months } \\
\hline $\mathrm{NIHSS}{ }^{5}$ median (IQR) & $0(1)$ & $1(1)$ & $0.5(3)$ & $0(1)$ & 0.122 \\
\hline $\mathrm{BI}^{5}$ median (IQR) & $100(0)$ & $100(0)$ & $95(17)$ & $100(0)$ & 0.017 \\
\hline mRS 5 median (IQR) & $1(1)$ & $2(1)$ & $3(2)$ & $1(1)$ & $<0.001$ \\
\hline Waist $^{2,3}$, mean cm (SD) & $95(13)$ & $89(15)$ & $98(15)$ & $95(13)$ & 0.137 \\
\hline Current smoking ${ }^{2,4}, \mathrm{n}(\%)$ & $31(9)$ & $1(7)$ & $4(17)$ & $26(9)$ & 0.597 \\
\hline \multicolumn{6}{|l|}{ Laboratory measures ${ }^{2,5}$} \\
\hline CRP, median (IQR), $n=267$ & $1.6(2.9)$ & $1.1(5.3)$ & $3.0(26.5)$ & $1.6(2.9)$ & 0.053 \\
\hline IL-6, median (IQR), $n=334$ & $4.6(4.5)$ & $4.8(4.1)$ & $6.1(6.1)$ & $4.2(4.2)$ & 0.038 \\
\hline Neopterin, median (IQR), $\mathrm{n}=334$ & $16(10)$ & $17(12)$ & $24(17)$ & $16(9)$ & $<0.001$ \\
\hline PAr-index, median (IQR), $n=334$ & $0.62(0.46)$ & $0.57(0.80)$ & $1.0(0.8)$ & $0.60(0.42)$ & 0.002 \\
\hline Kynurenic acid, $n=334$ & $58(30)$ & $56(35)$ & $65(52)$ & $59(29)$ & 0.522 \\
\hline
\end{tabular}

IS = ischemic stroke. TOAST = Trial of Org 10,172 in Acute Stroke Treatment. CHD = coronary heart disease. NIHSS=National Institute of Stroke Scale. IQR = interquartile range. $\mathrm{BI}=$ Barthels Index. $\mathrm{mRS}=$ modified Rankin Scale. $\mathrm{CRP}=\mathrm{C}$-reactive protein. IL-6=Interleukin-6. PAr-index $=$ pyridoxic acid ratio-index $=4$-pyridoxic acid:(pyridoxal+pyridoxal 5'-phosphate)

${ }^{1}$ Additionally, three patients died after having recurrent strokes

${ }^{2}$ At three months

${ }^{3}$ The one-way ANOVA was used for the continuous variables

${ }^{4}$ The chi-squared test was used for the categorical variables

${ }^{5}$ For the continuous variables with non-normal distribution, the non-parametric Kruskal-Wallis H-test was used

${ }^{6}$ TOAST subtype of index stroke

difference between the groups in the level of CRP, IL-6, and neopterin. Patients with cardioembolic stroke at baseline showed the highest values.

\section{Regression analyses}

The crude and adjusted hazard ratios (HR) for the outcome's ischemic stroke recurrence, death, and the composite endpoint of stroke and death, investigated for each of the biomarkers individually, are presented in
Table 3. We did not find any associations with stroke recurrence. In the crude analyses, all biomarkers except for KA were significantly associated with the risk of death or the composite endpoint. In the adjusted analyses, we found that CRP was associated with death (Model 1: HR 1.40, (CI 1.01, 1.96), $p=0.046)$ and neopterin with the combined endpoint (Model 1: HR 1.52, (CI 1.06, 2.20), $p=0.023$. Model 2: HR 1.55, (CI 1.06, $2.27), p=0.025)$. 
Table 2 Biomarker values at three-month follow-up by ischemic stroke subtype at baseline ${ }^{1}$

\begin{tabular}{|c|c|c|c|c|c|}
\hline & Atherosclerosis $(\mathrm{N}=26)$ & Cardioembolic $(N=62)$ & $\begin{array}{l}\text { CSVD } \\
(N=75)\end{array}$ & $\begin{array}{l}\text { Unknown } \\
(N=141)\end{array}$ & $P$-value \\
\hline $\mathrm{CRP}^{2}$, mean (SD) & $1.93(3.29)$ & $3.06(3.78)$ & $1.88(2.56)$ & $1.58(3.71)$ & 0.032 \\
\hline IL-6, mean (SD) & $3.71(1.95)$ & $5.70(2.18)$ & $3.97(2.18)$ & $4.26(2.39)$ & 0.038 \\
\hline Neopterin, mean (SD) & $16.0(1.5)$ & $20.3(1.7)$ & $16.0(1.4)$ & $16.0(1.5)$ & 0.003 \\
\hline PAr, mean (SD) & $0.66(1.77)$ & $0.73(1.73)$ & $0.60(1.73)$ & $0.60(1.56)$ & 0.087 \\
\hline KA, mean (SD) & $59.7(1.5)$ & $67.4(1.4)$ & $59.7(1.5)$ & $57.4(1.5)$ & 0.093 \\
\hline
\end{tabular}

${ }^{1}$ The category "other determined" was excluded because of low sample size. The analyses were conducted on log-transformed variables, and the values were transformed back after analysis

${ }^{2}$ For CRP: Atherosclerosis: $N=20$, cardioembolic: $N=46$, CSVD: $N=50$, Unknown: $N=100$. CSVD $=$ cerebral small vessel disease. CRP $=$ C-reactive protein IL-6 = Interleukin-6. PAr = pyridoxic acid ratio-index =4-pyridoxic acid:(pyridoxal+pyridoxal 5'-phosphate). KA=kynurenic acid. The analyses were done using a oneway ANOVA

In the additional competing risk regression analyses, where we used death as a competing event for stroke recurrence, the risk of ischemic stroke recurrence did not change significantly (see Supplementary Table 1).

The regression analyses, including both KA and neopterin in addition to the variables in Model 1, are shown in Table 4. KA was negatively associated with mortality (HR 0.57, (0.33, 0.97), 0.038), and neopterin was positively associated with mortality (HR $1.61,(1.02,2.54), 0.040)$. Neopterin was also associated with the composite endpoint (HR 1.59, (1.11, 2.27), 0.011).

Table 3 Crude and adjusted Cox regressions of the association between the individual biomarkers and ischemic stroke recurrence, death and the composite endpoint (ischemic stroke recurrence and death)

\begin{tabular}{|c|c|c|c|c|c|c|c|c|c|}
\hline & \multicolumn{3}{|c|}{ Crude } & \multicolumn{3}{|c|}{ Model 1} & \multicolumn{3}{|c|}{ Model 2} \\
\hline & HR & $\mathrm{Cl}$ & $P$ & HR & $\mathrm{Cl}$ & $p$ & HR & $\mathrm{Cl}$ & $P$ \\
\hline \multicolumn{10}{|c|}{ Recurrent ischemic stroke, $\mathrm{N}=16$} \\
\hline$\left(\mathrm{RP}^{1)}\right.$ & 0.90 & $(0.44,1.83)$ & 0.772 & 0.86 & $(0.41,1.77)$ & 0.674 & 0.92 & $(0.44,1.90)$ & 0.816 \\
\hline IL-6 & 1.11 & $(0.67,1.86)$ & 0.686 & 1.19 & $(0.52,1.97)$ & 0.578 & 1.10 & $(0.30,2.02)$ & 0.754 \\
\hline Neopterin & 1.34 & $(0.85,2.11)$ & 0.209 & 1.45 & $(0.82,2.57)$ & 0.201 & 1.39 & $(0.80,2.41)$ & 0.244 \\
\hline PAr & 1.16 & $(0.71,1.90)$ & 0.553 & 1.13 & $(0.61,2.10)$ & 0.692 & 1.24 & $(0.67,2.31)$ & 0.494 \\
\hline KA & 0.91 & $(0.55,1.50)$ & 0.710 & 0.82 & $(0.41,1.61)$ & 0.563 & 0.89 & $(0.43,1.86)$ & 0.761 \\
\hline \multicolumn{10}{|l|}{ Death $^{2}, N=28$} \\
\hline$\left(R^{1)}\right.$ & 1.81 & $(1.29,2.53)$ & 0.001 & 1.40 & $(1.01,1.96)$ & 0.046 & 1.41 & $(1.00,1.99)$ & 0.053 \\
\hline IL-6 & 1.74 & $(1.20,2.51)$ & 0.003 & 1.34 & $(0.87,2.07)$ & 0.181 & 1.36 & $(0.87,2.12)$ & 0.173 \\
\hline Neopterin & 1.93 & $(1.43,2.61)$ & $<0.001$ & 1.44 & $(0.89,2.33)$ & 0.136 & 1.55 & $(0.91,2.64)$ & 0.104 \\
\hline PAr & 1.69 & $(1.15,2.49)$ & 0.008 & 1.11 & $(0.68,1.82)$ & 0.666 & 1.13 & $(0.67,1.90)$ & 0.641 \\
\hline KA & 1.12 & $(0.75,1.66)$ & 0.577 & 0.64 & $(0.38,1.07)$ & 0.088 & 0.69 & $(0.41,1.14)$ & 0.145 \\
\hline \multicolumn{10}{|c|}{ Stroke and death, $N=41$} \\
\hline (RPP) & 1.59 & $(1.16,2.18)$ & 0.004 & 1.27 & $(0.92,1.74)$ & 0.140 & 1.27 & $(0.93,1.74)$ & 0.135 \\
\hline IL-6 & 1.47 & $(1.08,2.02)$ & 0.016 & 1.23 & $(0.85,1.77)$ & 0.274 & 1.23 & $(0.85,1.77)$ & 0.275 \\
\hline Neopterin & 1.79 & $(1.39,2.31)$ & $<0.001$ & 1.52 & $(1.06,2.20)$ & 0.023 & 1.55 & $(1.06,2.27)$ & 0.025 \\
\hline PAr & 1.62 & $(1.19,2.22)$ & 0.002 & 1.28 & $(0.86,1.90)$ & 0.224 & 1.34 & $(0.89,2.01)$ & 0.162 \\
\hline$K A$ & 1.27 & $(0.58,2.79)$ & 0.555 & 0.55 & $(0.19,1.54)$ & 0.252 & 0.60 & $(0.21,1.71)$ & 0.339 \\
\hline
\end{tabular}

$\mathrm{HR}=$ hazard ratio. $\mathrm{Cl}=$ confidence interval. $\mathrm{CRP}=\mathrm{C}$-reactive protein. IL- $6=$ Interleukin- 6 . $\mathrm{PAr}=\mathrm{PAr}$-index $=$ pyridoxic acid ratio-index $=4$-pyridoxic acid:(pyridoxal+pyridoxal-5'-phosphate). $\mathrm{KA}=$ kynurenic acid. Each biomarker was investigated individually

Model 1: Age, sex, prior cerebrovascular disease, modified Rankin Scale at 3 months, creatinine

Model 2: As in model $1+$ TOAST-classification at baseline (TOAST = Trial of Org 10,172 in Acute Stroke Treatment)

${ }^{1}$ For $C R P, N=212,8$ and 13 for all, recurrent IS and death, respectively

${ }^{2}$ Includes both those with and without ischemic stroke recurrence prior to death 
Table 4 Adjusted Cox regression (Model 1) of the combined contributions of kynurenic acid and neopterin in stroke, mortality and the composite endpoint (stroke and death)

\begin{tabular}{|c|c|c|c|c|c|c|c|c|c|}
\hline & Strol & urrence & & Deat & & & Strol & death & \\
\hline & HR & $\mathrm{Cl}$ & $p$ & HR & $\mathrm{Cl}$ & $p$ & HR & $\mathrm{Cl}$ & $p$ \\
\hline KA & 0.78 & $(0.39,1.57)$ & 0.492 & 0.57 & $(0.33,0.97)$ & 0.038 & 0.72 & $(0.46,1.10)$ & 0.131 \\
\hline Neopterin & 1.48 & $(0.83,1.57)$ & 0.177 & 1.61 & $(1.02,2.54)$ & 0.040 & 1.59 & $(1.11,2.27)$ & 0.011 \\
\hline Age & 0.98 & $(0.51,2.61)$ & 0.949 & 1.11 & $(0.60,2.06)$ & 0.746 & 1.09 & $(0.68,1.76)$ & 0.720 \\
\hline Sex & 1.39 & $(0.73,2.66)$ & 0.314 & 1.61 & $(1.00,2.62)$ & 0.052 & 1.43 & $(0.97,2.10)$ & 0.071 \\
\hline Prior CeVD & 1.37 & $(0.87,2.17)$ & 0.175 & 1.56 & $(1.10,2.22)$ & 0.012 & 1.44 & $(1.08,1.91)$ & 0.012 \\
\hline $\mathrm{mRS}$ & 0.97 & $(0.46,2.05)$ & 0.945 & 3.25 & $(1.77,5.96)$ & $<0.001$ & 2.24 & $(1.40,3.57)$ & 0.001 \\
\hline Creatinine & 1.31 & $(0.20,3.38)$ & 0.583 & 2.23 & $(1.12,4.42)$ & 0.022 & 1.65 & $(0.93,2.92)$ & 0.085 \\
\hline
\end{tabular}

The analyses includes KA, neopterin and Model 1: Age, sex, prior cerebrovascular disease, modified Rankin Scale at three months, creatinine

$\mathrm{HR}=$ hazard ratio. $\mathrm{Cl}=$ confidence interval. $\mathrm{KA}=$ kynurenic acid. $\mathrm{CeVD}=$ cerebrovascular disease. $\mathrm{mRS}=$ modified Rankin Scale

\section{Discussion}

In this study, we showed that higher levels of C-reactive protein (CRP) measured 3 months after acute ischemic stroke were associated with an increased risk of death. Neopterin was associated with the composite endpoint of stroke and death. In a model including both neopterin and kynurenic acid, neopterin and kynurenic acid were associated with an increased and a reduced risk of death, respectively. Furthermore, patients with cardioembolic stroke at baseline and those who died during follow-up had higher levels of pro-inflammatory biomarkers at 3 months.

\section{Stroke recurrence}

We did not find any association between inflammatory biomarkers and stroke recurrence. This could be due to low sample size, the relatively short time of follow-up, or not all recurrent strokes being clinically acknowledged leading to a statistical type II error. The role of inflammation in stroke recurrence, investigated by traditional biomarkers such as IL-6 and CRP, has been well established [14, 15, 33, 34]. The novel biomarkers; neopterin, PAr-index, and KA, are less studied. Neopterin has shown to predict future coronary events $[16,17]$ and stroke recurrence [35]. Neopterin has also been associated with complex carotid plaques in patients with stable angina pectoris [36], with the extent of cerebral small vessel disease (CSVD) in stroke patients [37] and with the presence, but not the future risk, of atrial fibrillation [38]. The PAr-index has been associated with future stroke in a population study [18] but has never been studied in a stroke population nor in the context of stroke recurrence. The role of KA in stroke recurrence is not clear, but KA has been found to be associated with coronary events [39, 40] and aortic stiffness, which in turn is associated with atrial fibrillation [41]. In a recent study, Baumgarten et al. found that the branch of the kynurenine pathway, leading to the formation of KA, was downregulated in atherosclerotic plaques, and this downregulation was associated with an increased risk of cerebrovascular events [19].

\section{Mortality}

We found that inflammation, in general, was significantly associated with mortality in the unadjusted analyses. In the adjusted analyses, only CRP and neopterin showed added value as predictors compared to traditional parameters such as prior vascular disease and $\mathrm{mRS}$ [30]. The lack of association to the remaining biomarkers could again be explained by statistical type II error due to the low sample size and short time of follow-up.

Several inflammatory biomarkers have been found to be independent predictors of all-cause and cardiovascular mortality. Both IL- 6 and CRP have been found to be associated with increased mortality [34, 42], and neopterin has been identified as an independent predictor of all-cause and cardiovascular mortality [16]. The PArindex has been associated with all-cause mortality in patients with coronary artery disease [43].

KA was negatively associated with mortality first after adjusting for inflammation. We did this analysis based on the increasing evidence of KA as a contributor to the body's anti-inflammatory capacity, as part of a negative feedback loop [19, 20, 44]. KA increases in response to inflammation, and our hypothesis, that a higher level of KA in response to inflammation, measured by neopterin [45] would be associated with a beneficial outcome, was supported as we found a higher value of KA to be associated with reduced risk of mortality when adjusted for inflammation. In fact, both risk estimates were strengthened after adjustment, indicating that they mutually camouflaged some of their individual contributions. 
Our current finding is in concordance with KA's role in vascular disease, amongst others, by inhibiting leukocyte recruitment to atherosclerotic plaques [19]. The result is in contrast to findings of KA being associated with mortality after coronary artery disease when being analysed without adjusting for inflammation [39]. Although exploratory, our results indicate that downregulation of KA might be one of the molecular pathways mediating the hazards of sedentary behaviour in disease progression. This should be further investigated.

\section{Stroke subtype}

We found that patients with cardioembolic strokes at baseline had higher levels of CRP, IL-6, and neopterin at 3 months, indicating a difference in the inflammatory profile between these subgroups. The link between stroke and inflammation has primarily been investigated in all the subtypes combined $[14,46]$ or in stroke caused by the progression of atherosclerosis [47] or CSVD [48]. The role of inflammation in cardioembolic strokes, in particular, is less studied. However, increasing evidence exists of the importance of inflammation for the presence of atrial fibrillation and the associated cardioembolic risk [7, 8, 49]. It can be argued that concurrent vascular disease might mediate the increased risk of stroke associated with inflammation found in patients with atrial fibrillation. However, Packer argued in a review for the contribution of inflammation as a risk factor independent of vascular disease, as the risk exceeded that predicted by cardiovascular risk factors [50]. Inflammatory biomarkers might be important for identifying those in need of prolonged ECG monitoring to identify paroxysmal atrial fibrillation, and when evaluating stroke risk in patients with atrial fibrillation [7, 38, 50-52]. Altogether, these observations indicate that the stroke subtype is relevant when studying inflammation in stroke and underlines the importance that such studies are powered for stratified analyses.

\section{Strength and weaknesses}

The information about stroke recurrence was based on the Norwegian Stroke Registry, which identifies patients with stroke admitted to hospital. The registry reports coverage of $84-87 \%$ in this period [26] and has been shown to be reliable [53]. The registry includes only patients admitted to hospital and where the recurrent stroke has been clinically recognised. Not all patients are admitted to hospital, and not all strokes are clinically overt. Zeestraten et al. found a recurrence rate of $3 \%$ based on clinical judgement, while $27 \%$ had new lacunas on imaging at the five-year follow-up [54]. These strokes might be more clinically subtle but are still of clinical importance. The use of imaging, such as MRI, could increase the sensitivity of the stroke diagnosis, and this has been included in the updated stroke definition [55]. Hence, we might have underestimated the incidence of ischemic stroke recurrence.

The mean follow-up time of the patients was 2.5 years, and for the patients included at the end of the study, the follow-up was limited to 16 months after the three-month follow-up. Khanevski et al. studied patients with ischemic stroke and transient ischemic attack and found that 5.4\% had stroke recurrence after 1 year, $10.7 \%$ after 5 years, and $14.2 \%$ at the end of the study (mean 5.6years) [30]. In addition, we had to exclude half of the stroke recurrences because they occurred before the three-month follow-up. The short follow-up and the low number of stroke recurrences in our study increase the risk of a type II error, and in particular negative findings should be evaluated with caution.

To avoid the acute inflammatory response associated with the acute stroke event, we chose the three-month follow-up after the acute stroke as the baseline for this study, assuming the inflammatory biomarkers were not affected by the acute event at that point in time-point [16]. The correlation between the inflammatory biomarkers before and 3 months after is not known. Still, it characterises these subgroups of patients and could be valuable for prospective studies. Stroke subtype is based on clinical judgement of the most likely cause of the stroke in question and may be challenging to determine [6]. Also, several causes of stroke in a single patient are common, i.e. a combination of large artery atherosclerosis, CSVD, and atrial fibrillation. These patients are often included in the category "unknown".

\section{Conclusion}

Neopterin seems to be a useful prognostic biomarker in stroke populations. The novel finding of a potential protective effect of KA in response to inflammation in disease progression should be investigated further. The results also underline the importance of stroke subtypes when investigating the impact of inflammation in ischemic stroke. The results highlights the importance of inflammation in cerebrovascular disease. Measures should be made to reduce inflammation as a secondary preventive strategy, i.e. by reducing sedentary behaviour. In addition, patients with undetected atrial fibrillation are receiving suboptimal drug treatment, and investigating the usefulness of inflammatory biomarkers to identify these patients could have significant therapeutic consequences for these individuals. 


\section{Abbreviations}

ATC: Anatomical therapeutic chemical classification; ADL: Activities of daily living; BI: Barthel Index; Cl: Confidence interval; CRP: C-reactive protein; CSVD: Cerebral small vessel disease; eGFR: Estimated glomerular filtration rate; ECG: Electro cardiogram; HR: Hazard ratio; IL-6: Interleukin-6; IL-10: Interleukin-10; IQR: Interquartile range; KA: kynurenic acid; LDL: Low density lipoprotein; mRS: Modified Rankin Scale; MRI: Magnetic resonance imaging; NIHSS: National Institute of Stroke Scale; Nor-COAST: Norwegian Cognitive Impairment After Stroke Study; PAr-index: Pyridoxal acid ratio index; PLP: Pyridoxal-5-phosphate; PL: Pyridoxal; PA: Pyridoxal acid; REK: regional ethics committee; SD: Standard deviation; TOAST: Trial of Org 10,172 in Acute Stroke Treatment; WHO: World Health Organization.

\section{Supplementary Information}

The online version contains supplementary material available at https://doi. org/10.1186/s12883-021-02498-w.

\section{Additional file 1:}

\section{Acknowledgements}

We want to thank the Nor-COAST research group and the dedicated staff at St Olavs hospital, Haukeland University hospital, Ålesund hospital, Vestre Viken Hospital Trust; Bærum Hospital, and Oslo University Hospital, Ullevål. We would also like to thank all participants and their relatives for volunteering for this project. The authors appreciate the valuable advice and practical assistance provided by expert personnel at Biobank $1^{\circledR}$, the research biobank of Central Norway, in collection, processing and storage of the biological material.

\section{Authors' contributions}

KNA has planned this study. She has participated in the processing of the registry data, planned and performed the statistical analyses and interpreted the results. She has written the article. AU has contributed to the statistical analyses and the interpretation of the results. He has contributed with detailed knowledge of the biomarkers from Bevital and to the process of writing the manuscript. TA has contributed to the design of this study and was responsible for the physical activity data from the main study. She has contributed to the revision of the manuscript. JA has supervised the process of developing and describing the statistical method and contributed to the interpretation and presentation of the data. TEM has analysed the cytokines used in this study. He has contributed in the post-analytical work and defined the method of handling the data and interpreting the results. He has also contributed in the revision of the manuscript. MN has contributed to the development of the study and revised the manuscript. HN has contributed to the development of the study and revised the manuscript. He has also contributed to the development of the statistical method and the interpretation of the results. IS is the principal investigator of the main project and has contributed to the revision of the manuscript. PMU has been in charge of analysing the biomarkers from Bevital. He has also contributed in the preparation phase of designing the study and selecting the analytic platform, and in interpreting the results and the revision of the manuscript. ABK has contributed to the development of the study and to the selection of biomarkers and interpretation of the results. She has also contributed to the writing process. All authors have read and agreed to the published version of the manuscript and are accountable for the content.

\section{Funding}

The lead author was granted a PhD-scholarship from the Western Norway Regional Health Authority, including funding for the laboratory analyses. The Nor-COAST study was granted by the Norwegian Health Association and the Norwegian University of Science and Technology (NTNU). The funders do not have an active role in the further scientific process.

\section{Availability of data and materials}

The datasets generated and/or analysed during the current study are not publicly available due to Norwegian regulations and conditions for informed consent but are available from the corresponding author on reasonable request.

\section{Declarations}

\section{Ethics approval and consent to participate}

The study was conducted in accordance with the institutional guidelines and approved by the Regional Committee of Medical and Health Research Ethics (REK) (Nor-COAST study, application number: REK no: 2017/2060. This substudy, application number: REK no: 2015/171, Application ID: 12253). The study was registered at Clinicaltrials.gov (NCT02650531). To participate, the patients had to be able to give written informed consent for themselves. Patients who were unable to express consent for themselves were also included if their next of kin did not decline. This is in line with the Norwegian consent procedures for patients not able to consent for themselves.

\section{Consent for publication}

Not applicable. The publication does not contain individual data.

\section{Competing interests}

$A B K$ has been/is the principal site investigator in three clinical trials (Boehringer-Ingelheim 1346.0023, Roche BN29553, and Novo Nordisk NN6535-4730), IS has been an investigator in the clinical trial BoehringerIngelheim 1346.0023 and part of the advisory board for Biogen. The rest of the authors do not have any competing interests to declare.

\section{Author details}

${ }^{1}$ Institute of Clinical Medicine (K1), University of Bergen, Bergen, Norway. ${ }^{2}$ Department of Internal Medicine, Haraldsplass Deaconess Hospital, Bergen, Norway. ${ }^{3}$ Bevital AS, Bergen, Norway. ${ }^{4}$ Department of Neuromedicine and Movement Science, Faculty of Medicine and Health Science, NTNUNorwegian University of Science and Technology, Trondheim, Norway. ${ }^{5}$ Centre for Clinical Research, Haukeland University Hospital, Bergen, Norway. ${ }^{6}$ Department of Immunology, Oslo University Hospital and University of Oslo, Oslo, Norway. ${ }^{7}$ Research Laboratory, Nordland Hospital, Bodø, and K.G. Jebsen TREC, University of Troms $\varnothing$, Troms $\varnothing$, Norway. ${ }^{8}$ Centre of Molecular Inflammation Research, NTNU-Norwegian University of Science and Technology, Trondheim, Norway. ${ }^{9}$ Department of Clinical Science (K2), University of Bergen, Bergen, Norway. ${ }^{10}$ Department of Neurology, Haukeland University Hospital, Bergen, Norway. ${ }^{11}$ Centre for age-related medicine, Stavanger University Hospital, Stavanger, Norway. ${ }^{12}$ Department of Geriatrics, Clinic of internal medicine, St Olavs Hospital, Trondheim University Hospital, Trondheim, Norway. ${ }^{13}$ Department of Geriatric Medicine, Oslo University Hospital. Ullevaal, Oslo, Norway.

Received: 24 September 2021 Accepted: 15 November 2021

Published online: 08 December 2021

\section{References}

1. Emerging Risk Factors C, Kaptoge S, Di Angelantonio E, Lowe G, Pepys MB, Thompson SG, et al. C-reactive protein concentration and risk of coronary heart disease, stroke, and mortality: an individual participant meta-analysis. Lancet. 2010;375(9709):132-40.

2. Parikh NS, Merkler AE, ladecola C. Inflammation, autoimmunity, infection, and stroke: epidemiology and lessons from therapeutic intervention. Stroke. 2020:51(3):711-8.

3. Dempsey PC, Matthews CE, Dashti SG, Doherty AR, Bergouignan A, van Roekel EH, et al. Sedentary behavior and chronic disease: mechanisms and future directions. J Phys Act Health. 2020;17(1):52-61.

4. Liberale L, Montecucco F, Schwarz L, Luscher TF, Camici GG. Inflammation and cardiovascular diseases: lessons from seminal clinical trials. Cardiovasc Res. 2021;117(2):411-22.

5. Adams HP Jr, Bendixen BH, Kappelle LJ, Biller J, Love BB, Gordon DL, et al. Classification of subtype of acute ischemic stroke. Definitions for use in a multicenter clinical trial. TOAST. Trial of org 10172 in acute stroke treatment. Stroke. 1993;24(1):35-41.

6. Rucker V, Heuschmann PU, O'Flaherty M, Weingartner M, Hess M, Sedlak C, et al. Twenty-year time trends in long-term case-fatality and recurrence rates after ischemic stroke stratified by etiology. Stroke. 2020;51(9):2778-85.

7. Berg DD, Ruff CT, Morrow DA. Biomarkers for risk assessment in atrial fibrillation. Clin Chem. 2021;67(1):87-95. 
8. Wallentin L, Hijazi Z, Andersson U, Alexander JH, De Caterina R, Hanna $M$, et al. Growth differentiation factor 15, a marker of oxidative stress and inflammation, for risk assessment in patients with atrial fibrillation: insights from the Apixaban for reduction in STroke and other THromboemboLic events in atrial fibrillation (ARISTOTLE) trial. Circulation. 2014;130(21):1847-58

9. Moore KJ, Sheedy FJ, Fisher EA. Macrophages in atherosclerosis: a dynamic balance. Nat Rev Immunol. 2013;13(10):709-21.

10. Poggesi A, Pasi M, Pescini F, Pantoni L, Inzitari D. Circulating biologic markers of endothelial dysfunction in cerebral small vessel disease: a review. J Cereb Blood Flow Metab. 2016;36(1):72-94.

11. Nasjonal faglig retningslinje - hjerneslag (National guidelines - stroke): Norwegian Directorate of Health; 2017 [updated 2020-04-27. Available from: https://www.helsedirektoratet.no/retningslinjer/hjerneslag.

12. Noz MP, Hartman YAW, Hopman MTE, Willems P, Tack CJ, Joosten LAB, et al. Sixteen-week physical activity intervention in subjects with increased cardiometabolic risk shifts innate immune function towards a less proinflammatory state. J Am Heart Assoc. 2019;8(21):e013764.

13. Alme KNA, T.; Assmus, J; Mollnes, T.E.; Naik, M.; Næss, H.; Saltvedt, l.; Ueland, P.M.; Ulvik, A.; Knapskog, A.B. Investigating novel biomarkers of immune activation and modulation in the context of sedentary behaviour: A multicentre prospective ischemic stroke cohort study. . BMC Neurology. 2021;in press.

14. Ridker PM, Everett BM, Thuren T, MacFadyen JG, Chang WH, Ballantyne C, et al. Antiinflammatory therapy with Canakinumab for atherosclerotic disease. N Engl J Med. 2017;377(12):1119-31.

15. Castillo J, Alvarez-Sabin J, Martinez-Vila E, Montaner J, Sobrino T, Vivancos $J$, et al. Inflammation markers and prediction of post-stroke vascular disease recurrence: the MITICO study. J Neurol. 2009;256(2):217-24.

16. Pedersen ER, Midttun O, Ueland PM, Schartum-Hansen H, Seifert R, Igland $J$, et al. Systemic markers of interferon-gamma-mediated immune activation and long-term prognosis in patients with stable coronary artery disease. Arterioscler Thromb Vasc Biol. 2011;31(3):698-704.

17. Sulo G, Vollset SE, Nygard O, Midttun O, Ueland PM, Eussen SJ, et al. Neopterin and kynurenine-tryptophan ratio as predictors of coronary events in older adults, the Hordaland health study. Int J Cardiol. 2013;168(2):1435-40.

18. Zuo H, Tell GS, Ueland PM, Nygard O, Vollset SE, Midttun O, et al. The PAr index, an indicator reflecting altered vitamin B-6 homeostasis, is associated with long-term risk of stroke in the general population: the Hordaland health study (HUSK). Am J Clin Nutr. 2018;107(1):105-12.

19. Baumgartner R, Berg M, Matic L, Polyzos KP, Forteza MJ, Hjorth SA, et al. Evidence that a deviation in the kynurenine pathway aggravates atherosclerotic disease in humans. J Intern Med. 2021;289(1):53-68.

20. Joisten N, Walzik D, Metcalfe AJ, Bloch W, Zimmer P. Physical exercise as kynurenine pathway modulator in chronic diseases: implications for immune and energy homeostasis. Int J Tryptophan Res. 2020;13:1178646920938688.

21. Thingstad P, Askim T, Beyer MK, Brathen G, Ellekjaer H, Ihle-Hansen $\mathrm{H}$, et al. The Norwegian COgnitive impairment after STroke (nor-COAST) study: study protocol of a multicentre, prospective cohort study. BMC Neurol. 2018;18(1):193.

22. Kuvas KR, Saltvedt I, Aam S, Thingstad P, Ellekjaer H, Askim T. The risk of selection bias in a clinical multi-center cohort study. Results from the Norwegian COgnitive impairment after STroke (nor-COAST) study. Clin Epidemiol. 2020;12:1327-36.

23. Pedersen ED, Waje-Andreassen U, Vedeler CA, Aamodt G, Mollnes TE. Systemic complement activation following human acute ischaemic stroke. Clin Exp Immunol. 2004;137(1):117-22.

24. Ulvik A, Midttun O, Pedersen ER, Eussen SJ, Nygard O, Ueland PM. Evidence for increased catabolism of vitamin B-6 during systemic inflammation. Am J Clin Nutr. 2014;100(1):250-5.

25. Midttun O, Hustad S, Ueland PM. Quantitative profiling of biomarkers related to B-vitamin status, tryptophan metabolism and inflammation in human plasma by liquid chromatography/tandem mass spectrometry. Rapid Commun Mass Spectrom. 2009;23(9):1371-9.

26. Norwegian Stroke Registry (Norsk Hjerneslagregister) - annual reports Norwegian Stroke Registry (Norsk hjerneslagregister); 2021 [Available from: https://stolav.no/fag-og-forskning/medisinske-kvalitetsregistre/ norsk-hjerneslagregister/rapporter.
27. Pedersen $A G$, Ellingsen CL. Data quality in the causes of death registry. Tidsskr Nor Laegeforen. 2015;135(8):768-70.

28. Fine JPG, R. J. A proportional hazards model for the subdistribution of a competing risk. J Am Stat Assoc. 1999;94(443):14.

29. Austin PC, Fine JP. Practical recommendations for reporting Fine-gray model analyses for competing risk data. Stat Med. 2017;36(27):4391-400.

30. Khanevski AN, Bjerkreim AT, Novotny V, Naess H, Thomassen L, Logallo $\mathrm{N}$, et al. Recurrent ischemic stroke: incidence, predictors, and impact on mortality. Acta Neurol Scand. 2019;140(1):3-8.

31. Mosarrezaii A, Amiri-Nikpour MR, Mehryar HR, Choobi Anzali B, NouroozZadeh S, Babaei S, et al. Investigating the relationship between interleukin-6 serum levels and outcome in acute ischemic CVA. Brain Behav. 2020;10(8):e01668.

32. Gu Y, Gutierrez J, Meier IB, Guzman VA, Manly JJ, Schupf N, et al. Circulating inflammatory biomarkers are related to cerebrovascular disease in older adults. Neurol Neuroimmunol Neuroinflamm. 2019;6(1):e521.

33. Everett BM, Glynn RJ, MacFadyen JG, Ridker PM. Rosuvastatin in the prevention of stroke among men and women with elevated levels of C-reactive protein: justification for the use of statins in prevention: an intervention trial evaluating Rosuvastatin (JUPITER). Circulation. 2010;121(1):143-50

34. Aulin J, Hijazi Z, Siegbahn A, Andersson U, Alexander JH, Connolly SJ, et al. Serial measurement of interleukin-6 and risk of mortality in anticoagulated patients with atrial fibrillation: insights from ARISTOTLE and RE-LY trials. JThromb Haemost. 2020;18(9):2287-95.

35. Ganz P, Amarenco P, Goldstein LB, Sillesen H, Bao W, Preston GM, et al. Association of osteopontin, neopterin, and myeloperoxidase with stroke risk in patients with prior stroke or transient ischemic attacks: results of an analysis of 13 biomarkers from the stroke prevention by aggressive reduction in cholesterol levels trial. Stroke. 2017;48(12):3223-31.

36. Sugioka K, Naruko T, Hozumi T, Nakagawa M, Kitabayashi C, Ikura Y, et al. Elevated levels of neopterin are associated with carotid plaques with complex morphology in patients with stable angina pectoris. Atherosclerosis. 2010;208(2):524-30.

37. Rouhl RP, Damoiseaux JG, Lodder J, Theunissen RO, Knottnerus IL, Staals $J$, et al. Vascular inflammation in cerebral small vessel disease. Neurobiol Aging. 2012;33(8):1800-6.

38. Lewicka E, Dudzinska-Gehrmann J, Dabrowska-Kugacka A, Zagozdzon P, Lizewska A, Danilowicz-Szymanowicz L, et al. Neopterin and interleukin- 6 as predictors of recurrent atrial fibrillation. Anatol J Cardiol. 2016;16(8):563-71.

39. Yu E, Ruiz-Canela M, Guasch-Ferre M, Zheng Y, Toledo E, Clish CB, et al. Increases in plasma tryptophan are inversely associated with incident cardiovascular disease in the prevencion con dieta mediterranea (PREDIMED) study. J Nutr. 2017;147(3):314-22.

40. Pedersen ER, Tuseth N, Eussen SJ, Ueland PM, Strand E, Svingen GF, et al. Associations of plasma kynurenines with risk of acute myocardial infarction in patients with stable angina pectoris. Arterioscler Thromb Vasc Biol. 2015;35(2):455-62.

41. Zapolski T, Kaminska A, Kocki T, Wysokinski A, Urbanska EM. Aortic stiffness: is kynurenic acid a novel marker? Cross-sectional study in patients with persistent atrial fibrillation. PLoS One. 2020;15(7):e0236413.

42. Hijazi Z, Aulin J, Andersson U, Alexander JH, Gersh B, Granger CB, et al. Biomarkers of inflammation and risk of cardiovascular events in anticoagulated patients with atrial fibrillation. Heart. 2016;102(7):508-17.

43. Ulvik A, Pedersen ER, Svingen GF, McCann A, Midttun O, Nygard O, et al. Vitamin B-6 catabolism and long-term mortality risk in patients with coronary artery disease. Am J Clin Nutr. 2016;103(6):1417-25.

44. Badawy AA. Hypothesis kynurenic and quinolinic acids: the main players of the kynurenine pathway and opponents in inflammatory disease. Med Hypotheses. 2018;118:129-38.

45. Pingle SK, Tumane RG, Jawade AA. Neopterin: biomarker of cell-mediated immunity and potent usage as biomarker in silicosis and other occupational diseases. Indian J Occup Environ Med. 2008;12(3):107-11.

46. Tardif JC, Kouz S, Waters DD, Bertrand OF, Diaz R, Maggioni AP, et al. Efficacy and safety of low-dose colchicine after myocardial infarction. N Engl J Med. 2019;381(26):2497-505.

47. Arenillas JF, Alvarez-Sabin J, Molina CA, Chacon P, Fernandez-Cadenas I, Ribo M, et al. Progression of symptomatic intracranial large artery atherosclerosis is associated with a proinflammatory state and impaired fibrinolysis. Stroke. 2008;39(5):1456-63. 
48. Noz MP, Ter Telgte A, Wiegertjes K, Joosten LAB, Netea MG, de Leeuw FE, et al. Trained immunity characteristics are associated with progressive cerebral small vessel disease. Stroke. 2018;49(12):2910-7.

49. Li Y, Tan W, Ye F, Wen S, Hu R, Cai X, et al. Inflammation as a risk factor for stroke in atrial fibrillation: data from a microarray data analysis. J Int Med Res. 2020;48(5):300060520921671.

50. Packer M. Characterization, pathogenesis, and clinical implications of inflammation-related atrial myopathy as an important cause of atrial fibrillation. J Am Heart Assoc. 2020;9(7):e015343.

51. Ma Y, Pan Z, Fan D, Xu S, Pan F. The increased risk of atrial fibrillation in inflammatory arthritis: a systematic review and meta-analysis of cohort studies. Immunol Investig. 2021:1-13.

52. Tancin Lambert A, Kong XY, Ratajczak-Tretel B, Atar D, Russell D, Skjelland $M$, et al. Biomarkers associated with atrial fibrillation in patients with ischemic stroke: a pilot study from the NOR-FIB study. Cerebrovasc Dis Extra. 2020;10(1):11-20

53. Varmdal T, Ellekjaer H, Fjaertoft H, Indredavik B, Lydersen S, Bonaa KH. Inter-rater reliability of a national acute stroke register. BMC Res Notes. 2015;8:584.

54. Zeestraten EA, Lawrence AJ, Lambert C, Benjamin P, Brookes RL, Mackinnon $A D$, et al. Change in multimodal MRI markers predicts dementia risk in cerebral small vessel disease. Neurology. 2017:89(18):1869-76.

55. Sacco RL, Kasner SE, Broderick JP, Caplan LR, Connors JJ, Culebras A, et al. An updated definition of stroke for the 21st century: a statement for healthcare professionals from the American Heart Association/American Stroke Association. Stroke. 2013;44(7):2064-89.

\section{Publisher's Note}

Springer Nature remains neutral with regard to jurisdictional claims in published maps and institutional affiliations.

- fast, convenient online submission

- thorough peer review by experienced researchers in your field

- rapid publication on acceptance

- support for research data, including large and complex data types

- gold Open Access which fosters wider collaboration and increased citations

- maximum visibility for your research: over $100 \mathrm{M}$ website views per year

At BMC, research is always in progress.

Learn more biomedcentral.com/submissions 